\title{
Feeding selectivity of introduced hedgehogs Erinaceus europaeus in a dryland habitat, South Island, New Zealand
}

\author{
Christopher Jones • Grant Norbury
}

Received: 14 December 2009 /Accepted: 7 September 2010/Published online: 20 November 2010

(C) Mammal Research Institute, Polish Academy of Sciences, Białowieża, Poland 2010

\begin{abstract}
We describe the diet of introduced European hedgehogs Erinaceus europaeus (Linnaeus, 1758) in a New Zealand dryland system and provide the first quantitative analysis of food selectivity for this species. We also describe and compare the diets of nine hedgehogs and measure dietary overlap between these individuals. The most commonly eaten foods were beetles, including rare native species (in 94\% of droppings), earwigs (92\%), spiders $(25 \%)$ and native skinks (14\%). Remains of at least three skinks were found in one dropping. Earwigs and darkling beetles (Tenebrionidae) were the most preferred food types, and Hymenoptera and cylindrical bark beetles (Colydiidae) were least preferred. Consumption of most foods mirrored their availability. Most individuals' diets conformed to the pattern seen at the population scale, with $21-54 \%$ of dry faecal mass derived from beetles and $13-$ $39 \%$ from earwigs. One animal frequently consumed large amounts of fruit. Dietary overlap between pairs of individuals was high (mean Horn's index, 0.84). This may be the result of limited opportunities to diversify in a very moisture-limited and low diversity habitat.
\end{abstract}

Communicated by: Andrzej Zalewski

C. Jones $(\bowtie) \cdot G$. Norbury

Landcare Research,

PO Box 282, Alexandra 9340, New Zealand

e-mail: jonesc@landcareresearch.co.nz

G. Norbury

e-mail: norburyg@landcareresearch.co.nz

Present Address:

C. Jones

Landcare Research,

PO Box 40, Lincoln 7640, New Zealand
Keywords Erinaceus europaeus · Diet · Dietary overlap · Food selection

\section{Introduction}

Information on a species' diet is vital in understanding its ecological relationships, particularly for a pest species, where it also allows an assessment of potential impacts on other ecosystem components (Litvaitis et al. 1996). European hedgehogs Erinaceus europaeus (Linnaeus, 1758) are native to much of western Europe and were introduced to New Zealand in the late nineteenth century, where they are now widespread and occupy many habitat types (Jones and Sanders 2005). Recent evidence has implicated hedgehogs as significant predators of endemic wildlife in island ecosystems into which they have been introduced, including New Zealand (Jackson and Green 2000; Sanders and Maloney 2002; Jones et al. 2005).

Most studies of hedgehog diet, in both New Zealand and Europe, have described consumption by hedgehogs in terms of the frequency of occurrence of a particular food type (e.g. Brockie 1959; Campbell 1973; Yalden 1976; Obrtel and Holišová 1981). This method is relatively robust for large sample sizes and enables comparisons between studies to be made, but the equal weight assigned to single and multiple items of different food types in a single sample can lead to biassed estimates of the relative importance of different foods to the consumer (Litvaitis et al. 1996). Accordingly, frequency-of-occurrence data alone can allow only limited inference as to whether foods are actively selected or avoided. Such information can only be obtained from comparisons of use with availability of different food types and is of particular importance when the consumer is an introduced pest that may be actively targeting rare prey of high 
conservation value. To the best of our knowledge, there have been no published accounts of food selectivity by hedgehogs.

Further understanding of the potential impacts of consumption by a pest species can come from studying the diets of individual animals. Although treating individuals as "average" representatives of a species greatly simplifies data collection and analysis, the inclusion of data concerning individual variation in feeding patterns may produce quite different model outcomes and management recommendations (Bolnick et al. 2003). Few studies have looked at individual diets (Fedriani and Kohn 2001; Sidorovich et al. 2001; Estes et al. 2003) even though the predatory behaviour of a small number of individual pests may cause significant impacts on prey of high conservation or economic value (Sacks et al. 1999; Anderson et al. 2002).

In this paper, we describe the diet of hedgehogs in a New Zealand dryland habitat and provide a quantitative analysis of food selectivity. We also describe and compare the diets of a small sample of individual animals and measure dietary overlap between these individuals.

\section{Study area}

Fieldwork was carried out in a 60-ha strip of dryland habitat bordered on one side by the true left bank of the Clutha (Matau-au) River, South Island, New Zealand $\left(45^{\circ} 15.85^{\prime} \mathrm{S}\right.$, $\left.169^{\circ} 23.52^{\prime} \mathrm{E}\right)$. Climate in the area is semi-arid continental and is characterized by relatively extreme seasonal and diurnal temperature ranges and low and unpredictable rainfall (annual mean, $360 \mathrm{~mm}$ ). Ground at the site rose $160 \mathrm{~m}$ from the river's edge (at $150 \mathrm{~m}$ a.s.l.) to a ridgeline some $300-400 \mathrm{~m}$ away. Adjacent to the river, vegetation was dominated by rank exotic grasses with stands of introduced willow (Salix fragilis) and poplar (Populus alba) lining the river bank. Introduced sweet briar (Rosa rubiginosa) and native matagouri (Discaria toumatou) shrubs were common. As the ground rose away from the river, ground cover became increasingly dominated by introduced thyme (Thymus vulgaris). Large boulders and rain-wash gullies were common on the slopes. At the upstream end of the site was a small irrigated orchard. Land use for the majority of the site was essentially recreational with a narrow walking path running parallel to the river.

\section{Material and methods}

Prey availability

Availability of hedgehog prey was estimated using 120 pitfall traps distributed randomly throughout the study site.
Each trap consisted of a 535-ml plastic food container (124 mm deep by $98 \mathrm{~mm}$ diameter) sunk into the ground so that the rim was flush with the ground surface. A small drainage hole in the bottom of each trap prevented accidental drowning of any captured McCann's skinks (Oligosoma maccanni Patterson and Daugherty, 1990) or invertebrates in the event of rain. Traps were protected with a raised wooden cover and left open for six nights in late March 2003. This was approximately the mid-point of the faecal sample collection period and is assumed to represent availability during the whole period. All captured invertebrates were identified, and a representative sample was preserved in alcohol for later use as a reference collection. Skinks were counted and released.

Hedgehog diets

Diet composition was investigated by analysis of hedgehog droppings. Faecal analysis has the advantages that study animals need not be killed and larger sample sizes are more easily obtainable compared with other methods that require killing of animals or collecting of carcasses (Fedriani and Kohn 2001). It is a commonly used method in the study of insectivore diets, including those of hedgehogs (Campbell 1973; Wroot 1984; Dickman and Huang 1988; Gilfillan 2001).

Diets were analysed at both individual and population level. To study the diets of individual hedgehogs, it was necessary to associate droppings with the animal producing them. Eleven animals were live-trapped in early February 2003. Captured hedgehogs were weighed and sexed, and a two-stage radio transmitter was attached by glueing it to a trimmed section of dorsal spines following Reeve (1982). Hedgehogs were radio-tracked to daytime nests at approximately weekly intervals between their initial date of capture and mid-April 2003. When traced to a nest, each animal was removed and placed in a cage overnight with nest material and adequate food and water. Any droppings produced were collected, and the animal was released at its capture location next morning. Some droppings were collected from nests, but these were only assigned to marked hedgehogs if they were clearly very fresh, as more than one hedgehog can use the same nest site (Reeve and Morris 1985). For population-scale analysis, droppings were collected opportunistically by observation whilst traversing the study site on foot from early February to mid-April 2003.

Of the 11 hedgehogs carrying radio transmitters, one male left the study area permanently and one was killed soon after the transmitter was attached. Between five and 12 faecal samples were obtained from each of the remaining nine animals. The smallest sample size was due to the hedgehog losing its transmitter for a period. This 
animal was later relocated and the transmitter reattached. The number of samples collected was limited by time and ethical constraints. We did not sample animals' diets more often than weekly because of concerns that this may disrupt their normal foraging and nesting behaviour. An unusually early onset of cold weather at the end of the study period also meant that some animals began to show hibernation behaviour, which caused the study to be concluded earlier than planned.

Overall, 137 droppings were collected, 77 of which were from known individuals and 60 from opportunistic collection. The dry mass of these samples ranged from 0.25 to $6.25 \mathrm{~g}$.

During the collection period, droppings were stored in a deep freeze. Prior to analysis, they were oven-dried to constant mass for $72 \mathrm{~h}$ at $50^{\circ} \mathrm{C}$ and weighed. After they were washed through a fine-meshed sieve, prey remains were viewed through a binocular dissecting microscope and identified using a variety of diagnostic structures (heads, elytra, legs, antennae, forceps). Invertebrate remains were generally identified to order, except for Coleoptera, which were identified to family and occasionally to species, using the reference collection and another collection of preserved local invertebrate fauna (courtesy of G.F. McLaren, HortResearch, Clyde, New Zealand). Vertebrate remains were readily identifiable, and each sample was scanned visually for the presence of earthworm chaetae.

Statistical analysis: population scale

All droppings collected were used to describe the diet and food selectivity of the local hedgehog population. For compatability with other studies, diet is described in terms of frequency of occurrence of each food type (equal to number of droppings containing a particular food type/total number of droppings). These data were then used with availability data from pitfall trapping to derive a preference index. We used Vanderploeg and Scavia's (1979) relativized electivity $E^{*}$, which Lechowicz (1982) found to be the best of seven tested: it has a zero value to indicate random feeding and a theoretical range of -1.0 to +1.0 . It is given by:

$E^{*}=\frac{[\mathrm{Wi}-(1 / n)]}{[\mathrm{Wi}+(1 / n)]}$,

where

$\mathrm{Wi}=\frac{r_{\mathrm{i}} / p_{\mathrm{i}}}{\sum_{\mathrm{i}}\left(r_{\mathrm{i}} / p_{\mathrm{i}}\right)}$,

given, $r_{\mathrm{i}}$ is the relative frequency of food type $\mathrm{i}$ in the diet and $p_{\mathrm{i}}$ is the relative frequency of food type $\mathrm{i}$ in the environment.
Individual diets

For the radio-tagged hedgehogs, the relative importance of different food types was investigated in greater detail using a predetermined scale based on that used by Lockie (1959) and Wise et al. (1981). The proportion of a dropping made up of each food type was estimated visually. An estimate of the dry mass of each food type in a dropping was obtained from the product of this proportion and the dry mass of the sample. These estimates for each food type were then summed for each individual and expressed as a percentage of the total faecal mass for each animal.

The proportions of the different food types in individuals' diets were compared using Friedmann's (1937) ANOVA on ranks. Whilst this test is less powerful than a standard parametric ANOVA, it is appropriate for data describing proportions. The degree of overlap between individuals' diets was compared using Horn's (1966) index which Krebs (1989) suggested as the best measure of overlap for data not expressed as frequencies.

$R_{\mathrm{o}}=\frac{\sum\left(p_{\mathrm{ij}} \pm p_{\mathrm{ik}}\right) \log \left(p_{\mathrm{ij}} \pm p_{\mathrm{ik}}\right)-\sum p_{\mathrm{ij}} \log p_{\mathrm{ij}}-\sum p_{\mathrm{ik}} \log p_{\mathrm{ik}}}{2 \log 2}$

where,

$R_{\mathrm{o}}=$ Horn's index of overlap for individuals $\mathrm{j}$ and $\mathrm{k}$

$p_{\mathrm{ij}}=$ proportion resource $\mathrm{i}$ is of total resources used by individual $\mathrm{j}$

$p_{\mathrm{ik}}=$ proportion resource $\mathrm{i}$ is of total resources used by individual $\mathrm{k}$.

\section{Results}

Diet of the local hedgehog population

The food types most commonly eaten by hedgehogs were beetles (Coleoptera), found in $94 \%$ of droppings, and earwigs (Dermaptera), in 92\% of droppings (Fig. 1). Detailed breakdown of beetle types showed that darkling (Tenebrionidae, 69\%), carabid (Carabidae, 63\%) and scarabaeid (Scarabidae, 54\%) beetles were the most commonly eaten. Remains of the locally restricted large sand scarab Pericoptus frontalis (Broun, 1904) and rare Alexandra chafer Prodontia modesta (Broun, 1909) were identified in $16 \%$ and $5 \%$ of droppings, respectively. Earwigs occurred in large numbers in most droppings, many of which consisted of at least $80 \%$ earwig remains, with one sample containing nothing else. Very small quantities of spider (Araneae) remains were found in $25 \%$ of droppings. These were mostly nocturnally active wolf spiders (Lycosidae). All identifiable Orthoptera remains 


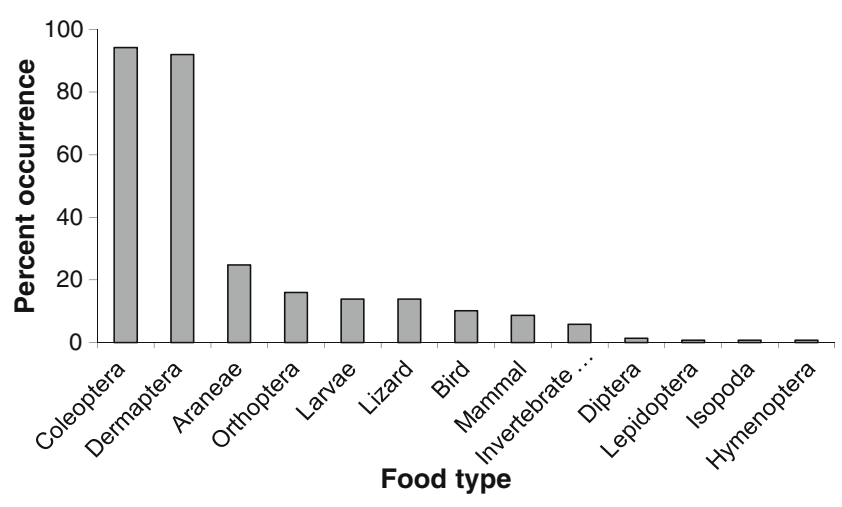

Fig. 1 Frequency of occurrence of food types in 177 hedgehog droppings from dryland habitat, South Island, New Zealand

were grasshoppers (Acrididae, 16\%), probably Phaulacridium marginale Walker, 1870. Larval remains (14\%) were often so macerated as to be unidentifiable.

Identifiable lizard remains (14\% of samples) ranged from a few scales to whole feet and bone fragments. One dropping contained the remains of at least 10 separate McCann's skink feet and another at least eight feet from the same species. One dropping contained two feet from the common gecko Hoplodactylus maculatus (Boulenger, 1885). Bird remains were found in $10 \%$ of droppings. These were mostly feathers with occasional skin remnants. Mammalian remains consisted of fur, some of which was still attached to skin. One sample contained a large number of small, probably mouse, bone fragments. Eight droppings obtained between 25 Feb. 2003 and 17 Mar. 2003 consisted almost entirely of invertebrate egg masses, which were probably molluscan in origin. Plant remains, generally of leaves and blades of grass, were seen in $49 \%$ of samples.

Estimation of selectivity was limited to those food types for which availability could be estimated using pitfall traps. Of these, earwigs and darkling beetles were the most preferred food types and Hymenoptera and cylindrical bark beetles (Colydiidae) were least preferred (Table 1).

\section{Individual diets}

Some foods contributed significantly more than others to individuals' diets (Friedmann chi-square $=7.02, \mathrm{df}=11, p<$ 0.001 ), but use of these foods did not vary significantly between individuals (Friedmann chi-square $=4.69, \mathrm{df}=8, p=$ 0.79 ). In general, food use conformed to the pattern seen at the population scale with heavy dependence on beetles (21$54 \%$ of the dry faecal mass of each animal) and earwigs (13$39 \%$ ). Bird and mammal remains made up over a quarter of the faecal mass of one male (L30) while another (T2/6) had large quantities of sweet briar seeds and fruit remnants ("rose hips") in five separate droppings, with over 100 seeds in a single dropping. This same animal had McCann's skink remains in five of nine $(56 \%)$ faecal samples. The next highest frequency of lizard remains was one out of seven samples (14\%). No lizard remains were found in any of up to 12 samples from five of the nine animals studied. Whilst these results (Table 2) suggest that some individuals may consume certain prey types more than other hedgehogs, low sample sizes meant that we had insufficient power to detect statistically significant variation between individuals' diets.

The degree of dietary overlap between individuals was generally high (Table 3 ). Mean overlap (36 pairs) was 0.84 (0.01 SE). The lowest index of overlap (0.66) was somewhat of an outlier with all others being greater than 0.72 . This indicates a very high level of dietary similarity between the animals studied.

\section{Discussion}

Diet of the hedgehog population

In this study, beetles were found in $94 \%$ of samples. This is a common finding in both European (Obrtel and Holišová 1981; Grosshans 1983; Wroot 1984) and some New Zealand studies (Berry 1999; Jones et al. 2005). Hedgehogs' heavy consumption of beetles may be of concern for the conservation values at our study site as two at-risk species were eaten. Both the large sand scarab beetle Pericoptus frontalis and the Alexandra chafer Prodontia modesta are considered to be vulnerable due to restricted range under the New Zealand Department of Conservation threat classification criteria (Molloy et al. 2002). Although both species occurred in relatively low frequencies in droppings, their restricted range means that sustained predation may have a significant impact on their viability.

Table 1 Vanderploeg and Scavia's (1979) relativized electivity $\left(E^{*}\right)$ values for a range of food types consumed by hedgehogs in dryland habitat, South Island, New Zealand

\begin{tabular}{lr}
\hline Food type & \multicolumn{1}{l}{$E^{*}$} \\
\hline Earwig & 0.3188 \\
Darkling beetle & 0.0209 \\
Scarab beetle & 0.0043 \\
Weevil & -0.0042 \\
Orthoptera & -0.0072 \\
Carabid beetle & -0.0079 \\
Lizard & -0.0081 \\
Spider & -0.0082 \\
Slaters (Isopoda) & -0.0082 \\
Hymenoptera & -0.0083 \\
Cylindrical bark beetle (Colydiidae) & -0.0083 \\
\hline
\end{tabular}


Table 2 Percentage of individual hedgehogs' dry faecal bulk made up by different food types as an index of diet composition
The category "other" includes grasses, leaf remnants, sand and fine gravels, and unidentifiable faecal matrix

\begin{tabular}{|c|c|c|c|c|c|c|c|c|c|c|c|}
\hline Hedgehog reference & L8 & L30 & L36 & $\mathrm{T} 2 / 1$ & $\mathrm{~T} 2 / 6$ & $\mathrm{~T} 2 / 5$ & $\mathrm{~T} 3 / 6$ & L18 & $\mathrm{L} 28$ & & \\
\hline Sex & M & $\mathrm{M}$ & $\mathrm{M}$ & $\mathrm{M}$ & M & $\mathrm{F}$ & $\mathrm{F}$ & $\mathrm{F}$ & $\mathrm{F}$ & & \\
\hline Mass (g) & 624 & 622 & 305 & 623 & 679 & 485 & 357 & 539 & 698 & & \\
\hline Number of samples & 9 & 8 & 5 & 9 & 9 & 10 & 12 & 8 & 7 & & \\
\hline Food type & \multicolumn{9}{|c|}{ Percent faecal bulk } & Mean & SE \\
\hline Coleoptera & 53 & 30 & 21 & 54 & 28 & 44 & 24 & 24 & 38 & 35.19 & 4.24 \\
\hline Dermaptera & 35 & 13 & 25 & 38 & 38 & 39 & 31 & 36 & 20 & 30.68 & 3.16 \\
\hline Other & 10 & 25 & 44 & 6 & 5 & 11 & 36 & 22 & 25 & 20.50 & 4.53 \\
\hline Mammalia & 0 & 12 & 0 & 0 & 0 & 0 & 5 & 12 & 10 & 4.29 & 1.81 \\
\hline Larvae & 0 & 2 & 9 & 0 & 3 & 3 & 1 & 0 & 2 & 2.25 & 0.88 \\
\hline Bird & 0 & 15 & 0 & 0 & 0 & 2 & 0 & 1 & 1 & 2.06 & 1.61 \\
\hline Fruit & 0 & 0 & 0 & 0 & 19 & 0 & 0 & 0 & 0 & 2.06 & 2.06 \\
\hline Lizards & 0 & 3 & 0 & 0 & 5 & 0 & 0 & 5 & 2 & 1.73 & 0.69 \\
\hline Araneae & 1 & 2 & 2 & 1 & 0 & 0 & 1 & 0 & 1 & 0.79 & 0.22 \\
\hline Orthoptera & 1 & 0 & 0 & 0 & 1 & 0 & 1 & 0 & 0 & 0.43 & 0.14 \\
\hline Diptera & 0 & 0 & 0 & 0 & 0 & 0 & 0 & 0 & 0 & 0.00 & 0.00 \\
\hline
\end{tabular}

Predation by hedgehogs on native lizards may also be of concern to conservation managers. Lizard remains were found in one in seven droppings in this study. The presence of 10 McCann's skink feet in one sample indicates that at least three skinks were eaten in a single feeding bout. Jones et al. (2005) noted a lower frequency of occurrence of skink remains in hedgehog guts than found here, but found a marked intersexual difference, with female hedgehogs three times more likely to prey on lizards than males.

Hedgehogs' ability to take full advantage of temporarily abundant foods is exemplified by the occurrence of large numbers of molluscan eggs in faecal samples obtained over a relatively short and well-defined period. Similar behaviour has been described in both New Zealand and Europe where hedgehogs took advantage of temporarily abundant grass grubs, cockchafer beetles (Amphimallon solstitialis Linnaeus, 1758) and aphid eggs (Campbell 1973; Obrtel and Holišová 1981; Wroot 1984).

Some studies have noted the importance of earthworms in hedgehog diets (Brockie 1959; Campbell 1973; Yalden
1976; Wroot 1984), but we found no evidence of earthworm chaetae to indicate consumption, nor were any earthworms seen when digging-in the 120 pitfall traps. The absence of earthworms was probably due to the very dry soil conditions at the time of the study. Earthworms require sufficient soil moisture to remain active near the soil surface where they would be accessible to hedgehogs.

\section{Diet selectivity}

Earwigs, the most preferred food in this study, were also considered a highly preferred food in Wroot (1984). The reason for this preference is unclear as earwigs have a relatively low energy content compared with other available foods (Wroot 1984; Reeve 1994). Possible explanations are that earwigs have nutritional value beyond their calorific content, that their smell or taste is particularly attractive to hedgehogs or that earwigs may represent a good "fallback" food source when drier conditions mean that soft-bodied prey are less abundant. Wroot (1984) noted a strong trend
Table 3 Degree of dietary overlap between individual hedgehogs

\begin{tabular}{|c|c|c|c|c|c|c|c|c|c|}
\hline Hedgehog reference & L8 & L18 & L28 & L30 & L36 & $\mathrm{T} 2 / 1$ & $\mathrm{~T} 2 / 5$ & $\mathrm{~T} 2 / 6$ & $\mathrm{~T} 3 / 6$ \\
\hline L8 & - & 0.85 & 0.87 & 0.76 & 0.81 & 0.99 & 0.97 & 0.83 & 0.92 \\
\hline L18 & & - & 0.95 & 0.88 & 0.84 & 0.84 & 0.87 & 0.79 & 0.95 \\
\hline L28 & & & - & 0.93 & 0.88 & 0.85 & 0.89 & 0.76 & 0.95 \\
\hline L30 & & & & - & 0.80 & 0.74 & 0.81 & 0.66 & 0.87 \\
\hline L36 & & & & & - & 0.77 & 0.85 & 0.72 & 0.94 \\
\hline $\mathrm{T} 2 / 1$ & & & & & & - & 0.97 & 0.84 & 0.83 \\
\hline $\mathrm{T} 2 / 5$ & & & & & & & - & 0.85 & 0.89 \\
\hline $\mathrm{T} 2 / 6$ & & & & & & & & - & 0.75 \\
\hline $\mathrm{T} 3 / 6$ & & & & & & & & & - \\
\hline
\end{tabular}

Overlap was estimated using Horn's (1966) index 
between earwig consumption and low rainfall and/or relative humidity, conditions which were manifest during our study.

Tenebrionid beetles were also a preferred prey, while carabid beetles were less so in spite of being a commonly eaten food in this and other studies. Carabids commonly employ chemical defences and are relatively fast moving, which may contibute to their being less preferred (Reeve 1994; Berry 1999). Although tenebrionids may also use chemical deterrents, they have a higher energy content per unit mass (Wroot 1984; Bell 1990), which may make them a more profitable prey.

Woodlice (Isopoda), Hymenoptera and cylindrical bark beetles were the least preferred foods. Woodlice have odour-producing lateral plate glands and contain relatively low levels of energy per unit mass, which may contribute to their low preference status in this and other studies of hedgehog diet (Dimelow 1963; Wroot 1984).

While ranking of foods, rather than absolute index values, is probably the most realistic indicator of preference (Lechowicz 1982; Norbury and Sanson 1992), in our study, there was an order of magnitude difference in $E^{*}$ values between earwigs and tenebrionid beetles and between the latter and all other food types. Most other foods had $E^{*}$ values of very close to 0 . This would suggest that selection or avoidance of these foods is very weak, i.e. hedgehogs in this environment consume foods in relation to their abundances. This may be the result of limited opportunities to diversify in a habitat that is very moisture-limited and where most of the ground cover is dominated by a single species ( $T$. vulgaris), thus limiting the complexity and diversity of the local terrestrial invertebrate community.

Both availability and use measures are subject to biasses that must be acknowledged. Pitfall trap results are biassed because actively moving surface fauna have a much higher probability of capture than sedentary forms, such as larvae (Cooper and Whitmore 1990). Numbers of individuals trapped allow inferences based on "activity-density" (Thiele 1977) which was considered a reasonable surrogate for availability in this study. Our pitfall traps were open continuously for 6 days so diurnally active species to which hedgehogs may not have had access could have been trapped. The potential for this was reduced by our use of dark wooden trap covers. Baars (1979) found that diurnally active species were caught less frequently in traps with dark covers than in those with light or transparent covers.

We also assumed that all local hedgehogs had equal access to all potential prey. Hedgehogs are non-territorial and show extensive intersexual and intrasexual home range overlap (Parkes 1975; Reeve 1982; Boitani and Reggiani 1984). Individuals in this study nested and were trapped throughout the study site.
In insectivore diet investigations using faecal analysis, variation in digestibility between hard- and soft-bodied prey types means that the latter are likely to be underrepresented, as are smaller prey items (Putman 1984; Dickman and Huang 1988). Prey remains are more broken down than in gut samples, and a large number of small fragments may be unidentifiable. In spite of these caveats, Dickman and Huang (1988) found good general agreement between ranked frequencies of taxa consumed and those recovered in droppings.

Individual diets

There was little individual variation in diet composition based on our analysis of faecal bulk contents. Only two animals' diets overlapped by a Horn's index value of less than 0.70: one male ate the smallest volume of earwigs, yet consumed the largest volume of bird and mammalian tissue, and another male was the only hedgehog to eat fruit. Some authors have dismissed the presence of fruit and other plant remains in hedgehog gut/faecal contents as resulting from accidental ingestion (Yalden 1976; Berry 1999), but this was clearly not the case here, with repeated, but temporally separate, samples containing large numbers of fruit. Other hedgehog species have been recorded deliberately eating fruit including Erinaceus amurensis (Schrenk, 1859) and Atelerix algirus, Lereboullet, 1842 (Liu 1937; Barquin et al. 1986 cited in Reeve 1994). We believe that the lack of individual variation in our studied animals' diets reflects the relatively harsh and resource-poor habitat and that incongruities such as heavy fruit consumption by one individual represent hedgehogs' ability to target energy-rich items that may become temporarily available. The non-selective feeding habit of hedgehogs means that they represent a significant threat to small isolated populations of prey, particularly where this species' own abundance is maintained by other food types.

Acknowledgements This study was funded by a Landcare Research Postdoctoral Fellowship. We are grateful to Gill McLaren and staff at HortResearch, Clyde, for their hospitality and use of their lab facilities. Guy Forrester provided statistical advice.

\section{References}

Anderson CR Jr, Ternent MA, Moody DS (2002) Grizzly bear-cattle interactions on two grazing allotments in northwest Wyoming. Ursus 13:247-256

Baars MA (1979) Catches in pitfall traps in relation to mean densities of carabid beetles. Oecologia 41:25-46. doi:10.1007/BF00344835

Bell GP (1990) Birds and mammals in an insect diet: a primer on diet composition analysis in relation to ecological energetics. Stud Avian Biol 13:416-422

Berry CJJ (1999) European hedgehogs (Erinaceus europaeus L.) and their significance to the ecological restoration of Boundary 
Stream Mainland Island, Hawke's Bay. MConservSci thesis, Victoria University of Wellington, New Zealand

Boitani L, Reggiani G (1984) Movements and activity patterns of hedgehogs (Erinaceus europaeus) in Mediterranean coastal habitats. Z Säugetierkd 49:193-206

Bolnick DI, Svanbäck R, Fordyce JA, Yang LH, Davis JM, Hulsey CD, Forister ML (2003) The ecology of individuals: incidence and implications of individual specialization. Am Nat 161:1-28. doi:10.1086/343878

Brockie RE (1959) Observations of the food of the hedgehog (Erinaceus europaeus L.) in New Zealand. NZ J Sci 2:121-136

Campbell PA (1973) The feeding behaviour of the hedgehog (Erinaceus europaeus L.) in pasture land in New Zealand. Proc NZ Ecol Soc 20:35-40

Cooper RJ, Whitmore RC (1990) Arthropod sampling methods in ornithology. Stud Avian Biol 13:29-37

Dickman CR, Huang C (1988) The reliability of fecal analysis as a method for determining the diet of insectivorous mammals. J Mammal 69:108-113. doi:10.2307/1381753

Dimelow EJ (1963) Observations on the feeding of the hedgehog (Erinaceus europaeus L.). Proc Zool Soc Lond 141:291-309. doi:10.1111/j.1469-7998.1963.tb01614.x

Estes JA, Riedman ML, Staedler MM, Tinker MT, Lyon BE (2003) Individual variation in prey selection by sea otters: patterns, causes and implications. J Anim Ecol 72:144-155. doi:10.1046/ j.1365-2656.2003.00690.x

Fedriani JM, Kohn MH (2001) Genotyping faeces links individuals to their diet. Ecol Lett 4:477-483. doi:10.1046/j.1461-0248.2001.00250.x

Friedmann M (1937) The use of ranks to avoid the assumption of normality implicit in the analysis of variance. J Am Stat Assoc 32:675-701. doi: $10.2307 / 2279372$

Gilfillan SL (2001) An ecological study of a population of Pseudantechinus macdonnellensis (Marsupialia: Dasyuridae) in central Australia. I. Invertebrate food supply, diet and reproductive strategy. Wildl Res 28:469-480

Grosshans W (1983) Zur Nahrung des Igels (Erinaceus europaeus L. 1758). Untersuchungen von Magen-Darminhalten schleswigholsteinischer Igel. Zool Anzeig Jena 211:364-384

Horn HS (1966) Measurement of "overlap" in comparative ecological studies. Am Nat 100:419-424. doi:10.1086/282436

Jackson DB, Green RE (2000) The importance of the introduced hedgehog (Erinaceus europaeus) as a predator of the eggs of waders (Charadrii) on machair in South Uist, Scotland. Biol Conserv 93:333-348. doi:10.1016/S0006-3207(99)00135-4

Jones C, Sanders M (2005) European hedgehog. In: King CM (ed) The handbook of New Zealand mammals, 2nd edn. Oxford University Press, Auckland, pp 81-94. doi:10.1016/j.mambio.2007.05.002

Jones C, Moss K, Sanders M (2005) Diet of hedgehogs (Erinaceus europaeus) in the upper Waitaki Basin, New Zealand: implications for conservation. NZ J Ecol 29:29-35

Krebs CJ (1989) Ecological methodology. Harper Collins, New York

Lechowicz MJ (1982) The sampling characteristics of electivity indices. Oecologia 52:22-30. doi:10.1007/BF00349007
Litvaitis JA, Titus K, Anderson EM (1996) Measuring vertebrate use of terrestrial habitats and food. In: Bookhout TA (ed) Research and management techniques for wildlife and habitats, 5th edn. The Wildlife Society, Bethesda, pp 254 274

Liu C-C (1937) Notes on the food of Chinese hedgehogs. J Mammal 18:355-357. doi: $10.2307 / 1374212$

Lockie JD (1959) The estimation of the food of foxes. J Wildl Manage 23:224-227. doi: $10.2307 / 3797647$

Molloy J, Bell B, Clout M, de Lange P, Gibbs G, Given D, Norton D, Smith N, Stephens T (2002) Classifying species according to threat of extinction. A system for New Zealand. Threatened Species Occas Publ 22. Department of Conservation, Wellington

Norbury GL, Sanson GD (1992) Problems with measuring diet selection of terrestrial, mammalian herbivores. Aust $\mathrm{J}$ Ecol 17:1-7. doi:10.1111/j.1442-9993.1992.tb00774.x

Obrtel R, Holišová V (1981) The diet of hedgehogs in an urban environment. Folia Zool 30:193-201

Parkes J (1975) Some aspects of the biology of the hedgehog (Erinaceus europaeus L.) in the Manawatu, New Zealand. NZ J Zool 2:463-472. doi:10.1080/03014223.1975.9517887

Putman RJ (1984) Facts from faeces. Mammal Rev 14:79-97. doi:10.1111/j.1365-2907.1984.tb00341.x

Reeve NJ (1982) The home range of the hedgehog as revealed by a radio tracking study. Symp Zool Soc Lond 49:207-230

Reeve NJ (1994) Hedgehogs. T. and A.D. Poyser, London

Reeve NJ, Morris PA (1985) Construction and use of summer nests by the hedgehog (Erinaceus europaeus). Mammalia 49:187-194. doi:10.1515/mamm.1985.49.2.187

Sacks BN, Jaeger MM, Neale JCC, McCullough DR (1999) Territoriality and breeding status of coyotes relative to sheep predation. J Wildl Manage 63:593-605. doi:10.2307/3802648

Sanders MD, Maloney RF (2002) Causes of mortality at nests of ground-nesting birds in the upper Waitaki Basin, South Island, New Zealand: a 5-year video study. Biol Conserv 106:225-236. doi:10.1016/S0006-3207(01)00248-8

Sidorovich VE, MacDonald DW, Pikulik MP, Kruuk H (2001) Individual feeding specialization in the European mink, Mustela lutreola and the American mink, M. vison in north-eastern Belarus. Folia Zool 50(1):27-42

Thiele H-U (1977) Carabid beetles in their environments. SpringerVerlag, Berlin

Vanderploeg HA, Scavia D (1979) Calculation and use of selectivity coefficients of feeding: zooplankton grazing. Ecol Model 7:135149. doi:10.1016/0304-3800(79)90004-8

Wise MH, Linn IJ, Kennedy CR (1981) A comparison of the feeding biology of mink Mustela vison and otter Lutra lutra. J Zool, Lond 195:181-213. doi:10.1111/j.1469-7998.1981.tb03458.x

Wroot AJ (1984) Feeding ecology of the European hedgehog, Erinaceus europaeus. $\mathrm{PhD}$ thesis, University of London, UK

Yalden DEW (1976) The food of the hedgehog in England. Acta Theriol 21:401-424 